(FECa) were used to indicate renal dysfunction. Urinary $\mathrm{Cd}$ was used as exposure marker.

Results We found that high FECa was related to high bone fracture risk in both genders. Proximal tubular dysfunction and glomerular dysfunction did not relate to bone fracture risk.

Conclusion Abnormal Cd handling was a key risk factor for bone fracture in $\mathrm{Cd}$ exposed people. Men was at risk of bone fracture risk as similar as women. FECa was a specific indicator of $\mathrm{Ca}$ wasting and its determination cost was cheaper compare to $\beta_{2}-\mathrm{MG}$ and serum cystatin $\mathrm{C}$. We recommended to use FECa to monitor abnormal $\mathrm{Ca}$ metabolism and individual who showed high FECa should have a special health promotion to reduce bone fracture risk. Reduced renal toxicant exposure, and $\mathrm{Ca}$ supplementation were suggested as a health promotion for this Cd exposed farmer.

\section{GENETICS AND EPIGENETICS IN TOXICOLOGY OF METALS}

${ }^{1}$ Karin Broberg, ${ }^{2}$ Roberto G Lucchini. 'Institute of Environmental Medicine, Karolinska Institutet, Stockholm, Sweden; ${ }^{2}$ Occupational Medicine, University of Brescia, Italy

\subsection{6/oemed-2018-ICOHabstracts. 1441}

Aim of special session To provide current knowledge on genetic and epigenetic effect of metal exposure in different population.

\section{4a EPIGENETIC ALTERATIONS AS AN UNDERLYING MECHANISM OF THE TOXICITY OF METALS}

\footnotetext{
1,2Karin Broberg. 'Institute of Environmental Medicine, Karolinska Institutet, Stockholm, Sweden; ${ }^{2}$ Department of Laboratory Medicine, Lund University, Lund, Sweden
}

\subsection{6/oemed-2018-ICOHabstracts. 1442}

Introduction Toxic metals are present at many workplaces as well as in food and drinking water, causing life-long daily exposure. In the last decade, epigenetic alterations have gained attention as underlying mechanisms of the adverse health effects observed in relation to exposure to metals. Epigenetic marks, broadly defined as dynamic changes to the genome other than changes in the DNA sequence itself, can lead to persistent changes in the regulation of gene expression. Epigenetic mechanisms have key functions in regulating cellular homeostasis, and lineage-specific gene expression, and perturbations may have long-lasting influence on future health, including risk of cancer.

Methods Epigenetic changes in candidate genes and in the epigenome (pyrosequencing, MS-HRM, target-enrichment nextgeneration sequencing, RNA sequencing, histone modifications, and microRNA) have been measured in different tissues in relation to exposure to toxic metals (arsenic, cadmium, lead, welding particles) in samples collected from humans occupationally and/or environmentally exposed to metals.

Result We have shown that exposure to particularly arsenic, but also cadmium and metal-containing particles, are significantly associated with changes of epigenetic marks, and some of these marks are linked to carcinogenesis, cardiovascular and immune function. The epigenetic changes seem to persist over several years and for arsenic and cadmium sex differences were found.

Discussion Research during the last decade have shown that metals common at workplaces and in the general environment are associated with epigenetic alterations that in turn may have long-lasting effects on the gene regulation and risk of disease. Experimental and animal studies support that epigenetic perturbations are underlying mechanism for metal toxicity. Similar to what has been shown for smoking-related epigenetic marks, metal-related epigenetic marks may be promising exposure and effect biomarkers.

\section{4b MANGANESE TOXICITY ACROSS THE LIFESPAN FROM PRENATAL EXPOSURE TO THE OLD AGE}

${ }^{1,2}$ Roberto G Lucchini. 'Occupational Medicine, University of Brescia, Italy; ${ }^{2}$ Occupational Medicine, Icahn School of Medicine at Mount Sinai, New York, USA

\subsection{6/oemed-2018-ICOHabstracts. 1443}

Introduction Manganese exposure causes neurotoxicity from prenatal life to the old age. An extended body of literature is now available on:

i. different exposure sources and routes of absorption;

ii. mechanistic aspects provided also by imaging assessment;

iii. dose-response relationship from epidemiological and animal studies;

iv. gender differences;

v. genetic predisposition.

Methods New evidence was reviewed from the scientific literature and the studies presented during the world conference held in 2016 at the Icahn School of Medicine at Mount Sinai, New York, USA.

Results Current manganese research offers a wide spectrum of observations within a continuum of exposure from early life to old age, with new evidence of neurotoxicity at low exposure levels in occupational settings and operations including welding. Gender differences are consistently shown by human studies and toxicity vs beneficial effects are reported to varying as a function of the exposure windows. Brain imaging studies through structural and functional MRI provide new information about differences and similarities between occupational, environmental exposure and parkinsonian conditions. Genetic studies focused on genes regulating specific transporters, such as SLC30A10, offer new prospective in understanding the regulation of metabolic pathway and toxico-dinamic properties of manganese.

Conclusion Manganese exposure is increasingly common through a variety of industrial and agricultural products. Current research offers consistent evidence of potential links from neurodevelopment to neurodegeneration. The enhanced vulnerability of specific exposure windows and the cumulative mechanism of toxicity require further studies to identify the critical windows of susceptibility and the exposure levels suitable to protect the brain throughout the lifetime exposure. Understanding these aspects is of outmost relevance in view of the potential role of manganese as determinant of both neurodevelopmental disorders and neurodegenerative diseases. 